neofilolog

Czasopismo Polskiego Towarzystwa Neofilologicznego

ISSN 1429-2173, elSSN 2545-3971, 2020, NR 54/1, 87-104

http://dx.doi.org/10.14746/n.2020.54.1.6

http://poltowneo.org/

\title{
Monika Janida
}

Uniwersytet Marii Curie-Skłodowskiej w Lublinie https://orcid.org/0000-0002-5603-4803 monika.janicka@poczta.umcs.lublin.pl

\section{DAS POTENZIAL KOLLEGIALEN FEEDBACKS FÜR DIE FÖRDERUNG DER EVALUATIONSKOM PETENZ BEI ANGEHENDEN FREM DSPRACHENLEHRENDEN - ERGEBNISSE EINER STUDIE}

\section{The potential of peer-to-peer-feedback for enhancing assessment competence in pre-service language teachers - results of a study}

The article introduces formative assessment as one of the most powerful factors influencing students' achievements and presents its significance for process-oriented learning and teaching. Due to its importance formative assessment should be an inherent part of the pre-service teacher training program. The aim of the present paper is to highlight the potential of peer-to-peer-feedback, as a part of formative assessment, for enhancing the assessment competence of pre-service teachers. The results of the study presented in the article suggest ways in which peer-to-peer feedback can be used to foster the assessment competence of prospective teachers.

Keywords: formative assessment, peer-to-peer-feedback, assessment competence, pre-service teacher training

Słowa kluczowe: ocenianie kształtujące, ocenianie koleżeńskie, kompetencja diagnostyczna, kształcenie nauczycieli 


\section{Einleitende Überlegungen}

Die Erkenntnisse der Neurowissenschaften sowie der kognitiven und konstruktivistischen Didaktik und Pädagogik haben neues Licht auf den Zugang zu Rollen und Aufgaben für Lehrpersonen und Schülerinnen und Schüler geworfen: Da Lernen ein selbstgesteuerter, eigenwilliger und eigensinniger Prozess ist, der zwar Informationen, Anregungen, Rückmeldungen und Lernhilfen braucht, sich aber nicht von außen determinieren lässt (Siebert, 2005: 32), sollten die Rollen der Lehrpersonen und der Lernenden neu definiert werden. Die soziale Umgebung spielt dabei eine wichtige Rolle, denn „soziales Lernen ist ein zirkuläres Verhältnis, das die Zurechnungsfähigkeit von vornherein in eine antizipierte Wirkung der eigenen Handlungen im Blick auf die Wahrnehmung durch andere und eigene Reaktionen hierauf richtet" (Reich, 2004: 172). Im konstruktivistisch geprägten Unterricht müssen Lehrende in einer doppelten Rolle handeln: Zum einen müssen sie mehrwissende Experten sein, zum anderen als "lerner orientierte M oderatoren der Wissens- und Handlungskonstruktion" agieren (Reich, 2004: 205). Damit die Wissens- und Handlungskonstruktion gefördert werden können, müssen Handlungsspielräume für zunehmend entdeckendes, problemlösendes, handlungso rientiertes, selbstverantwortetes, selbstständiges und kooperatives Lernen geschaffen werden. Diese methodischen Grundprinzipien erfordern Aufgabenformate, die ein hohes Maß an Autonomie und Mitbestimmung seitens der Lernenden zulassen. Solche Anforderungen erfüllen sog. offene Aufgaben wie Freiarbeit, Wochen planarbeit, projektorientierter Unterricht, Stationenarbeit, kooperative Lernformen oder Werkstattunterricht. Offene Unterrichtsformen setzen zugleich einen neuen Leistungsbegriff und neue Formen der Leistungsbewertung voraus. Um der Prozesshaftigkeit des Lernens gerecht zu werden, müssen Formen von Beurteilung und Kontrolle in Anspruch genommen werden, die sich nicht lediglich auf Produkte von Leistungsaktivitäten beziehen, wo aufbereitetes Wissen zu einem vorgegebenen Zeitpunkt in Form von Tests abgefragt wird, sondern die ein komplexes und differenziertes Förder- und Beratungsverfahren berücksichtigen. Das ermöglicht zum Beispiel die Schülerselbst- und Mitbewertung (Bohl, 2004: 16), die wiederum auf neue Facetten der Evaluationskompetenz der Lehrperson hinweist.

Diagnostizieren, förderndes Beurteilen ${ }^{1}$ sowie Beraten haben starken Einfluss auf die Effizienz der Lernprozesse, was empirisch nachgewiesen werden konnte. Fördernde Bewertung steht auf der Liste von Faktoren, die sich am stärksten auf die Lernprozesse auswirken, mit dem Effekt 0,90 an 4. Stelle, während Feedback mit dem Effekt von 0,75 an 10. Stelle steht (Hattie, 2015:

${ }^{1}$ Auch als formative Beurteilung bezeichnet (vgl. Czaplikowska, Kubacki, 2019: 29). 
367). Im prozessorientierten Unterricht sollten die Leistungen der Lernenden auch der Lehrperson als Ausgangspunkt zur Reflexion dienen, wie man den Unterricht entwickeln und somit die Qualität der Bildungsprozesse erhöhen kann (Bohl, 2004: 79-81). Da Bewerten und Beurteilen der Leistungen eine bedeutende Rolle bei der Förderung der Lernprozesse spielen, sollte die Entwicklung einer Evaluationskompetenz ein inhärenter Bestandteil des Trainings von angehenden Fremdsprachenlehrerinnen und -lehrern sein.

\section{Vorbereitung angehender Lehrkräfte auf förderdiagnostische Aufgaben}

Traditionelle Leistungsbewertung soll hauptsächlich Aufschluss darüber geben, ob und inwiefern die Lernenden die im Bildungscurriculum festgesetzten Lernziele erreicht haben. In dieser Hinsicht ist sie selektionsorientiert. In der fördernden Bewertung, die dem pädagogischen Leistungsbegriff verpflichtet ist, werden nicht nur die finalen Leistungen der Lernenden beurteilt, sondern innen wird vor allem Auskunft darüber gegeben, wie ihr Lernprozess abläuft: was sie bereits erreicht haben und woran sie noch arbeiten müssen. Diese Art Bewertung ist persönlichkeitsfördernd und setzt eine vertrauensvolle Beziehungsstruktur voraus (Bohl, 2004: 27). Die Lernenden bekommen konkrete Hilfen, die sie beim Erreichen ihrer Ziele unterstützen (Sterna, 2016: 6). Fördernde Bewertung hilft den Lernenden, Antworten auf drei Fragen zu finden: „Wohin gehe ich?", „Wie gehe ich dorthin?" und „Wie komme ich weiter?". Um diese Fragen beantworten zu können, brauchen die Lernenden klare Kriterien, die festlegen, was und auf welche Weise sie erreichen sollen, und die innen helfen, sich weitere anspruchsvolle Ziele zu setzen. Effektives Feedback vollzieht sich auf mehreren Ebenen: Auf der Ebene der Aufgabenbearbeitung wird das Feedback oft als Korrektur bezeichnet (corrective feedback) und gibt den Lernenden eine Rückmeldung, ob sie die Aufgabe korrekt gelöst haben. Diese Korrektur beschränkt sich aber meistens auf nur eine konkrete Lösung und ist auf andere Aufgaben nicht übertragbar. Sie kann effektiv sein, wenn sie die Lernenden über ihre falschen Annahmen und Interpretationen aufklärt. Auf der Prozessebene hilft das Feedback, die eigenen Fehler zu entdecken und angemessene Strategien beim Lösen der Aufgabe einzusetzen. Diese Art Rückmeldung ermöglicht tieferes Lernen. Auf der Ebene der Handlungsregulierung fördert das Feedback die Autonomie der Lernenden, denn es ermöglicht den Einsatz von kognitiven Lernstrategien, schafft Voraussetzungen für die Selbstevaluation und fördert somit die Selbstregulierung (Hattie, Timperley, 2007: 90-96).

Effektives Feedback kann auch auf dem dreistufigen FSE-M odell ${ }^{2}$, Fakten - Stellungnahme - Erwartungen, basieren. Fakten beziehen sich auf konkrete,

${ }^{2}$ Auf Polnisch heißt dieses M odell FUO: Fakty - Ustosunkowanie - Oczekiwania. 
beobachtbare Situationen, die stattgefunden haben (Ich habe beobachtet, dass ...). In der Stellungnahme äußert man seine subjektive, in der 1. Person Singular formulierte Einstellung zu Fakten (Ich finde, dass...). Im dritten Schritt formuliert die Lehrperson ihre Erwartungen und Lösungsvorschläge (Ich würde mir wünschen / Es wäre besser....) (vgl. Kordziński, 2013: 293)3.

Bildungsstandards für angehende Lehrkräfte berücksichtigen die Thematik der Evaluation in unterschiedlichem $\mathrm{M}$ aße. In den Bildungsstandards für Lehrkräfte aus dem Jahr $2012^{4}$ werden folgende Aspekte der Evaluation thematisiert, die angehenden Lehrenden im Laufe ihrer Ausbildung beigebracht werden sollen: Kontrolle und Beurteilung der Leistungen von Schülerinnen und Schülern, Konstruieren von Tests, Arten der Bewertung, kontinuierliche Bewertung, Semesterbewertung, Jahresbewertung, interne und externe Bewertung, Funktionen von Bewertung, externe Abschlussprüfung. ${ }^{5}$

In der aktuellen Verordnung des Polnischen M inisters für Wissenschaft und Hochschulbildung ${ }^{6}$, die ab August 2019 neue Bildungsstandards zur Ausbildung von Lehrenden festlegt, wird der Förderung der diagnostischen Kompetenz zukünftiger Lehrkräfte etwas mehr Platz eingeräumt. Die förderdiagnostischen Kompetenzen auszubildender Lehrender verbleiben zwar hauptsächlich auf der Wissensebene, umfassen aber neben der Kenntnis der traditionellen Bewertungsformen auch den pädagogischen Leistungsbegriff, der sowohl Fachkompetenzen als auch den Lernprozess sowie die Persönlichkeitsentwicklung von Schülerinnen und Schülern unterstützen soll. ${ }^{7}$

\footnotetext{
${ }^{3}$ M ehr zu Methoden der fördernden Bewertung ist bei Sterna (2016) zu finden.

4 http://prawo.sejm.gov.pl/isap.nsf/download.xsp/WDU20120000131/0/D20120131.pdf [DW $=8.10 .2019$ ]

${ }^{5}$ „2.2.9-2.3.9. Kontrola i ocena efektów pracy uczniów. Konstruowanie testów i sprawdzianów. Ocenianie i jego rodzaje. Ocenianie bieżące, semestralne i roczne. Ocenianie wewnętrzne i zewnętrzne. Funkcje oceny. Sprawdzian kończący etap edukacyjny" (S. 13-14). ${ }^{6}$ http://isap.sejm.gov.pl/isap.nsf/download.xsp/WDU20190001450/0/D20191450.pdf [DW $=8.10 .2019$ ]

${ }^{7}$ Absolwent zna i rozumie: D.1/E.1.W10. rolę diagnozy, kontroli i oceniania w pracy dydaktycznej; ocenianie i jego rodzaje: ocenianie bieżące, semestralne i roczne, ocenianie wewnętrzne i zewnętrzne; funkcje oceny; D.1/E.1.W11. egzaminy kończące etap edukacyjny i sposoby konstruowania testów, sprawdzianów oraz innych narzędzi przydatnych w procesie oceniania uczniów w ramach nauczanego przedmiotu; D.1/E.1.W12. diagnozę wstępną grupy uczniowskiej i każdego u cznia w kontekście nauczanego przedmiotu lub prowadzonych zajęć oraz sposoby wspomagania rozwoju poznawczego uczniów; potrzebę kształtowania pojęć, postaw, umiejętności praktycznych, w tym rozwiązywania problemów, i wykorzystywania wiedzy; metody i techniki
} 


\section{Didaktisches Forschungsprojekt - Ziele, Methoden, Durchführung}

Das Projekt, das im Rahmen eines Methodik-Kurses an der Marie-Curie-Skłodowska-Universität im Sommersemester 2018/2019 durchgeführt wurde, diente der Förderung der Lehrkompetenzen und insbesondere der Reflexions- und Evaluationskompetenzbei angehenden Lehrpersonen. Ein weiteres Ziel, das mit dem Projekt verfolgt wurde, war der Vertiefung des Wissens über die Förderung von Schülerinnen und Schülern mit besonderem Förderbedarf im Fremdsprachenunterricht gewidmet. Das Forschungsprojekt wurde mit Studierenden des dritten Semesters des M A-Studiums mit Englisch als erster Fremdsprache und Deutsch, Französisch oder Russisch als zweiter Fremdsprache im Rahmen von zwei Kursen durchgeführt: M ethodik der zweiten Fremdsprache (30 Unterrichtsstunden) und Kompensationsdidaktik - Arbeit mit Schülern mit besonderen Förderbedürfnissen im Fremdsprachenunterricht (15 Unterrichtsstunden).

\subsection{Ablauf des Projektes}

Am Anfang des Kurses wurde den Studierenden das FSE-M odell (siehe oben), das beim Strukturieren der fördernden Bewertung sehr hilfreich sein kann, vorgeführt. Die Studierenden wurden dafür sensibilisiert, dass die traditionelle Bewertung es beim Formulieren von kritischen Bemerkungen und dem Hinweisen auf Unzulänglichkeiten bewenden lässt, während die fördernde Bewertung einen Schritt weiter geht und an die kritischen Bemerkungen Änderungsvorschläge anschließt. Betont wurde dabei, dass die Lehrperson die Bewertung der Schülerleistungen zugleich als Feedback über die Qualität ihres Unterrichts wahrnehmen und als wichtigen Hinweis betrachten sollte, an den eigenen Unterrichtsmethoden zu feilen.

Es wurde bereits erwähnt, dass die konstruktivistische Didaktik, die sich die Entwicklung von Autonomie und M it- und Selbstbestimmung bei Schülerinnen und Schülern zum Ziel setzt, eine ganz andere Feedbackkultur und neue Instrumente zum Bewerten der Schülerleistungen braucht. In dem Projekt wurden andere Studierende in den Bewertungsprozess einbezogen; sie haben im Rahmen des Trainings die kollegiale M itbewertung eingeübt. In der ersten Trainingsphase wurden den Studierenden Unterrichtsmitschnitte präsentiert ${ }^{8}$,

skutecznego uczenia się; metody strukturyzacji wiedzy oraz konieczność powtarzania i utrwalania wiedzy i umiejętności; (...) (S. 18).

${ }^{8}$ Die Sequenz stammt aus Ziebell, B. (2007), Unterrichtsbeobachtung und Lehrerverhalten. Fernstudieneinheit 32. Videosequenzen. Berlin: Langenscheidt. Obwohl die ausgewählte Publikation bereits etwas verstaubt ist, hat sie den Vorteil, dass die darin 
über die sie sachliche Rückmeldungen geben und zu denen sie Änderungsvorschläge formulieren sollten.

In der zweiten Phase sollten die Studierenden in Kleingruppen kurze Unterrichtssequenzen von 20-25 M inuten durchführen. Es handelte sich dabei um Simulationen von Situationen aus dem Anfängerunterricht, die jeweils ein kommunikatives Ziel verfolgten, das auf Deutsch, Französisch oder Russisch umgesetzt wurde. Dabei sollten Unterrichtsmethoden ausgewählt und Unterrichtsmaterialien vorbereitet werden, die Lernenden mit besonderen Förderbedürfnissen entgegenkamen und ihre Lernprozesse unterstützten. Während der Simulation haben sich ausgewählte Studierende in die Rolle des Förderbedürftigen versetzt. In jeder Unterrichtssequenz sollten jeweils andere Förderbedürfnisse im Fokus stehen: Legasthenie, ADHS, Seh-und Hörstörungen, geistige Behinderung, das Asperger-Syndrom sowie Hochbegabung ${ }^{9}$. Die Studierenden wurden im Vorfeld auf die Problematik des Inklusionsunterrichts aufmerksam gemacht (Al-Khamisy, 2013; Janicka, 2016; Janicka, 2017; Reich, 2014). Sie sollten ihr Wissen darüber vertiefen, wie sich besondere Förderbedürfnisse auf den Lernprozess auswirken und sich über Unterrichtsmethoden und -materialien informieren, die den Lernprozess von Schülern mit diesen Schwierigkeiten fördern und ihren Bedürfnissen und Begrenzungen gerecht werden. Im Anschluss an die durchgeführte Unterrichtssequenz sollten die Studierenden einen interaktiven Vortrag vorbereiten, in dem sie explizit auf die besonderen Förderbedürfnisse der jeweiligen Zielgruppe eingehen und die Wahl der Unterrichtsmethoden begründen.

In der Vorbereitungsphase wurde gemeinsam mit den Studierenden ein Bewertungsbogen entwickelt. Klare und verständliche Bewertungskriterien, die mit Hilfe der Likert-Skala ausgewertet werden sollten, dienten dazu, den Teilnehmenden des Projektes eine objektive und vielseitige Bewertung der beobachteten Unterrichtssequenzen zu erleichtern. Der Fragebogen umfasste zahlreiche Aspekte, die im M ethodik-Kurs thematisiert und trainiert wurden, wie Unterrichtsplanung, die Wahl von aktivierenden Unterrichtsmethoden und Sozialformen oder die Unterrichtssprache. Darin wurde auch die Anpassung von Unterrichtsmethoden und $M$ aterialien an die Möglichkeiten von Lernenden mit besonderen Förderbedürfnissen berücksichtigt.

präsentierten Sequenzen komplexe Unterrichtsabläufe und nicht lediglich punktuelle, thematisch profilierte Unterrichtsabschnitte darstellen. Es wurde angenommen, den Projektteilnehmenden dürfte es leichter fallen, sich damit kritisch auseinander zu setzen.

${ }^{9}$ Diese Förderbedürfnisse wurden aufgrund der einschlägigen Fachliteratur und der Angaben des polnischen Hauptamtes für Statistik (GUS) ausgewählt, weil sie bei Schülern von öffentlichen Schulen relativ häufig vorkommen. 
Die oben genannten Aspekte wurden durch folgende Indikatoren erfasst:

1. Berücksichtigung der Sprachkompetenz und Möglichkeiten der Lernenden: Kreativität bei der Entwicklung von Aufgaben und Materialien/Engagieren aller Teilnehmenden/Guter Kontakt mit den Teilnehmenden/Anpassung des Schwierigkeitsgrades an die M öglichkeiten der Gruppe/Kommunikation in der Zielsprache/Struktur der Unterrichtssequenz/gleichmäßige Entwicklung von verschiedenen Sprachfertigkeiten;

2. Berücksichtigung besonderer Förderbedürfnisse: Anpassung des Unterrichtstempos/Anpassung der Unterrichtsmaterialien/Lehrerverhalten (Sprechtempo, Augenkontakt, Körpersprache etc.), abgestimmt auf die M öglichkeiten der Lernenden/Berücksichtigung verschiedener Lernstile/Ermöglichung kognitiven, emotionalen und sozialen Lernens;

3. Interaktiver Vortrag/Form: Aktivierung aller Teilnehmenden/Engagement aller Präsentierenden;

4. Interaktiver Vortrag/Inhalt: Vorstellung von Schlussfolgerungen zur Förderung von Lernenden/Präsentation von Wissen über besondere Förderbedürfnisse/gute Vorbereitung der Gruppe auf ihre Expertenrolle.

Die Indikatoren wurden mithilfe der vereinfachten Likert-Skala ausgewertet: stimme gar nicht zu (1) - stimme eher nicht zu (2) - stimme eher zu (3) stimme entschieden zu $(4)^{10}$.

\subsection{Ergebnisse des Trainings zur fördernden Bewertung}

An dem Training waren 19 Studierende beteiligt, die in sieben Gruppen sieben Unterrichtssequenzen durchgeführt haben. Nach jeder Präsentation wurden die Bewertungsbögen anonym ausgefüllt, indem Punkte mithilfe der LikertSkala vergeben wurden. Die Beurteilenden wurden explizit darum gebeten, neben den Punkten auch inre Kommentare (auf Polnisch) zu dem beobachteten Unterrichtsgeschehen abzugeben. Die evaluierte Gruppe nahm, nachdem sie sich mit den Evaluationsergebnissen vertraut gemacht hatte, zu der Bewertung im Plenum Stellung. Die Aufgabe hatte zum Ziel, die Reflexionsfähigkeit sowie das Formulieren von und den Umgang mit konstruktiver Kritik zu fördern.

Die Auswertung ergab 94 Auswertungsbögen. Maximal konnten von jeder Gruppe 72 Punkte erreicht werden. Im Folgenden werden ausgewählte

\footnotetext{
$10 \mathrm{Um}$ dem Ausweichen auf die neutrale Mittelkategorie vorzubeugen, wurde eine vierstufige, geradzahlige Antwortskala benutzt. Angesichts der oft abgemilderten, ausweichenden Aussagen der Befragten (was bei der Auswertung der Bewertungsbögen deutlich wurde) erwies sich diese Entscheidung als richtig (vgl. Bortz; Döring, 2003: 179).
} 
Kommentare präsentiert, die sich auf die Unterrichtssequenzen beziehen, die am besten (70 Punkte) und am schwächsten (61,3 Punkte) beurteilt wurden.

Die kritischen Kommentare zu Gruppe 6 (mit dem schwächsten Ergebnis) beziehen sich darauf, dass die Unterrichtenden vorwiegend frontal gehandelt haben, ohne die ganze Gruppe zu engagieren:

„Die einzige kritische Bemerkung betrifft die Tatsache, dass ihr nicht alle engagiert habt. Im Grunde genommen hat nur eine Person die Fragen zu den Aufgaben beantwortet" $[\mathrm{Fb} \mathrm{9}]^{11}$.

"(...) ich würde mir persönlich wünschen, dass jeder Teilnehmende die Chance hätte, sich in der Fremdsprache zu äußern“ [Fb 7] $]^{12}$.

Die Kommentare zum lernerzentrierten und engagierenden Unterricht widersprechen sich aber, denn in einem anderen Fragebogen [Fb 4] wird gelobt, dass die ganze Gruppe aktiviert worden sei.

Die zu hohen Ansprüche, die an die Gruppe gestellt wurden, wurden ebenfalls zur Sprache gebracht:

"(...) meiner M einung nach war der Stoff ein bisschen zu schwierig, denn ihr habt das ganze Alphabet eingeführt und sehr viele Bezeichnungen für Bekleidung. (...) Bei der Präsentation von Buchstaben herrschte an der Tafel ziemlich viel Unordnung [Fb 4$]^{13}$.

Der Fokus dieser Unterrichtssequenz sollte auf der Arbeit mit Schülerinnen und Schülern mit dem Asperger-Syndrom liegen. In dieser Hinsicht wurden gewisse $M$ ängel thematisiert:

"Die Präsentation über AS war okay, aber ich habe konkrete Informationen vermisst, wie man Unterrichtsmaterialien (an die Bedürfnisse) dieser Schüler anpassen soll“ $[\mathrm{Fb} \mathrm{5}]^{14}$.

11 "Jedyną uwagą jest to, że prowadzący nie zaangażowali wszystkich uczestników (nawet odpowiedzi do zadań nie są podawane przez wszystkich, tylko przez jedną osobę)". Übersetzung aller Kommentare durch die Autorin.

12 "(...) osobiście wolałbym, aby każdy uczestnik miał okazję indywidualnie wypowiedzieć się w obcym języku".

13 „(...) moim zdaniem troszeczkę za trudny materiał, bo w prowadziliście i cały alfabet i sporo nazw ubrań. (...) Po drugie przy zapisywaniu liter na tablicy był troszeczkę nieporządek".

${ }^{14}$ (...) prezentacja problemu była ok co do samego Zespołu Aspergera, ale zabrakło mi w niej konkretnych informacji co do tego jak należy dostosować materiały edukacyjne dla ucznia. 
Andere Kommentare, die sich aber auf die gleichen Aspekte (M enge an eingeführter Lexik sowie Erklärungen zur Arbeit mit Schülern mit dem AspergerSyndrom) bezogen, waren jedoch äußerst positiv:

„Vernünftige Portion an Wortschatz" [Fb 2] ${ }^{15}$

„Sehr interessanter und dynamisch durchgeführter Unterricht. Ihr habt die ganze Gruppe dank vielfältiger Übungstypen engagiert. Ihr habt nur Russisch gesprochen und eine Person aus der Gruppe um die Übersetzung gebeten " $[\mathrm{Fb} 4]^{16}$.

"(...) toll, dass ihr den Unterschied zwischen Autismus und Asperger-Syndrom erklärt habt" $[\mathrm{Fb} 6]^{17}$.

„Eine sehr interessante Themenwahl, hervorragende Präsentation und große Auswahl an Aufgaben. Der Unterricht wurde völlig an die Bedürfnisse des Schülers mit dem Asperger-Syndrom angepasst" ${ }^{4}[\mathrm{Fb} 7]^{18}$.

Die sich teilweise ausschließenden Bewertungen derselben Unterrichtsaspekte können zum einen von großer Subjektivität bei der Wahrnehmung zeugen. Zum anderen aber muss betont werden, dass einige Personen in den Fragebögen zugaben, Russisch über mehrere Jahre hinweg gelernt zu haben. Eben für diese Personen dürfte diese Unterrichtssequenz nicht überfordernd gewesen sein, was eine Begründung für die widersprüchlichen Kommentare sein könnte.

Die Gruppe 2, die ebenfalls eine Unterrichtssequenz auf Russisch durchgeführt hat, bekam die höchste Bewertung. Die Kommentare, die sich auf die Unterrichtsführung und Hinweise zur Arbeit mit gehörgeschädigten Schülerinnen und Schülern bezogen, waren überwiegend positiv:

„(...) die Lehrpersonen haben die Präsentation über Gehörgeschädigte in der sehr attraktiven Form eines Quiz durchgeführt. Dadurch wurde die ganze Gruppe zum M itmachen und zur Diskussion aktiviert" [Fb 89] ${ }^{19}$.

\footnotetext{
${ }_{15}^{15}$ "przystępna porcja słownictwa"

${ }_{11}^{16}$ Bardzo ciekawa i dynamicznie poprowadzona lekcja. Zaangażowaliście całą grupę przygotowując różnorodne zadania. Używaliście tylko j. obcego, a o wyjaśnienie tego, co mówiliście, prosiliście osobę z grupy".

${ }^{17}$ "(...) fajnie, że pokazaliście różnice między zespołem Aspergera a autyzmem".

${ }_{18}^{18}$ "Bardzo ciekawy dobór tematu i wspaniała prezentacja materiału, także znakomity dobór ćwiczeń. Cała lekcja została w pełni dostosowana do specjalnej potrzeby edukacyjnej". 19 "Prowadzące przeprowadziły prezentację SPE w bardzo pomysłowej formie quizu, co pozwoliło zaktywizować całą grupę do pracy i dyskusji".
} 
„Es hat mir sehr gefallen. Ihr habt die Unterrichtsmethoden an die Bedürfnisse der ganzen Klasse und die Bedürfnisse des förderbedürftigen Schülers geschickt angepasst" [Fb 90] ${ }^{20}$.

„Die Idee, Namensschilder in kyrillischer Schrift zu verteilen, finde ich toll. Ihr habt qualitätsvolle Unterrichtsmaterialien vorbereitet; der neue Wortschatz wurde auf verschiedene Weisen eingeführt und eingeübt. M einer Meinung nach war der Unterricht sehr engagierend und interessant " [Fb 86] ${ }^{21}$.

"(...) Ich glaube, alle Elemente guten Unterrichts wurden berücksichtigt und ich fand nichts, was mir an diesem Unterricht gefehlt hätte" [Fb 85] ${ }^{22}$.

"(..) der Unterricht war an die Bedürfnisse von Schülern mit Gehörschaden angepasst. Guter Kontakt zwischen den Lehrpersonen und der Gruppe. Angemessene Portion an Fremdsprache und M uttersprache - alles verständlich. Interessante Aktivitäten (...." ${ }^{\prime \prime}[\mathrm{Fb} 92]^{23}$.

Es gab einige wenige kritische Bemerkungen, die weitere Aspekte der beobachteten Unterrichtssequenz ansprachen:

„M ein einziger Einwand ist, dass die Aussprache etwas mehr geübt werden könnte und es könnte mehr individuelle Wiederholungen geben“ [Fb 88 $]^{24}$.

Allerdings gab es bei dem Aspekt der Ausspracheschulung widersprüchliche Bewertungen desselben Unterrichtsgeschehens:

„Es war zu sehen, dass ihr euch sehr gut vorbereitet habt. Ihr habt die Aussprache korrigiert, was ein großer Vorteil ist. (...)" [Fb 93 ${ }^{25}$.

Analysiert man die Evaluationsbögen, so fällt ins Auge, dass ein Teil der Kommentare sehr präzise ausgewählte Aspekte der Unterrichtsführung anspricht,

${ }^{20}$ „Dobrze połączyłyście potrzeby całej klasy z potrzebami ucznia o specjalnych potrzebach edukacyjnych".

${ }^{21}$ "Pomysł z imionami zapisanymi cyrylicą - świetny! Przygotowałyście bardzo dobre materiały, nowe słownictwo zostało opracowane i przećwiczone na wiele sposobów". ${ }_{22}^{22}$ "Wydaje mi się, że wszystko zostało zawarte i nie mogę znaleźć żadnej rzeczy, której by mi brakowało w tej lekcji".

${ }^{23}$ ()...) lekcja dobrze dostosowana do potrzeb ucznia z niedosłuchem. Dobry kontakt prowadzących z grupą. Odpowiednia ilość języka obcego i polskiego - wszystko zrozumiałe. Ciekawe aktywności (...)"

${ }^{24}$ "Jedyne moje zastrzeżenie jest takie, że troszkę większy nacisk mógł być położony na wymowę, troszeczkę więcej powtórzeń indywidualnie".

${ }^{25}$ "Widać, że obie bardzo dobrze się przygotowałyście. Zwracałyście uwagę na wymowę, co jest bardzo dużym plusem. (...)". 
ein Teil aber auf einer Allgemeinheitsebene verbleibt. Die Kommentare sollten nicht nur positive und negative Beobachtungen wiedergeben, sondern nach dem FSE-M odell auch Hinweise darauf liefern, was man hätte anders tun können, damit die Unterrichtsziele auf eine effizientere Weise hätten erreicht werden können. Eine genaue Analyse ergab, dass lediglich 18 Fragebögen (etwa $20 \%$ der Bewertungen) Verbesserungsvorschläge enthielten, selbst wenn die Kritik an der durchgeführten Unterrichtssequenz in Form von reduzierter Punktevergabe auf der Likert-Skala geäußert wurde. Die Wahl bestimmter, sich wiederholender Ausdrücke bei Änderungsvorschlägen wie ein bisschen ("troszkę większy nacisk" [Fb 88], „troszeczkę więcej powtórzeń, „zabrakło mi troszeczkę bardziej klarownego wyjaśnienia odmiany haben“ [Fb 24], „można by troszeczkę bardziej zwolnić tempo [Fb 35], „troszkę więcej nacisku położyłbym na wymowę" [Fb 66]), das Einzige (,jedyne, co dodałabym [Fb 40], "Jedyne, co bym zmieniła“ [Fb 26], [Fb 56], „pomyślałabym jedynie“ [Fb 50], "Jedyne moje zastrzeżenie jest takie [Fb88]), schade, dass („szkoda, że nie przećwiczyliśmy wymowy [Fb 62]), unpersönliches man könnte („M ożna było dodatkowo rozwinąć ten temat i dodać więcej zwrotów" [Fb 77], „zadania były za łatwe, można było dodać coś więcej” [Fb70], „można by powiedzieć dokładnie, co będzie robione" [Fb 94]) sowie vielleicht („może jakaś inna forma aktywizacji byłaby lepsza" [Fb 70]), könnte davon zeugen, dass die Studierenden die Kritik an Kommilitoninnen und Kommilitonen aus der Gruppe scheuten und ihre Empfehlungen, die das Ergebnis kritischer Beobachtungen waren, abmildern wollten. Das wiederum legt den Schluss nahe, dass in diesem Bereich noch Diskussions- und Trainingsbedarf besteht.

\subsection{Forschungsinstrument}

In dem Kernteil der Studie sollten die Studierenden mithilfe eines Auswertungsbogens einschätzen, inwiefern sich ihre Reflexions- und Evaluationskompetenz durch die Teilnahme an dem didaktischen Projekt entwickelt haben. Abgegeben wurden 18 Bewertungsbögen. Sie erfassten Aspekte, wie die motivierende Rolle der fördernden Bewertung, die Entwicklung der Lehrkompetenz oder die Entwicklung der diagnostischen Förderkompetenz. Die Umfrage sollte Antwort auf folgende Forschungsfragen liefern:

1. Inwiefern hat die Mitbewertung durch Studierende zur Entwicklung der Lehrerkompetenz beigetragen?

2. Inwiefern hat das Projektkonzept zur Entwicklung der Kompetenz beigetragen, mit Schülern mit besonderen Förderbedürfnissen im Fremdsprachenunterricht zu arbeiten? 
3. Inwiefern hat die Teilnahme am Projekt zur Entwicklung der Förderkompetenz bei angehenden Fremdsprachenlehrenden beigetragen?

Frage Nummer 1 wurde durch folgende Items erfasst: die Bewertung und Kommentare in den Auswertungsbögen waren für mich sehr motivierend - dank der Bewertungen und Kommentare gewann ich mehr Selbstsicherheit bei der Unterrichtsführung - dank der Bewertungen und Kommentare zu meiner Präsentation weiß ich, worauf ich achten soll und was ich in meinen Unterrichtsmethoden verändern soll - dank der Bewertungen und Kommentare zu meiner Präsentation konnte ich meine Stärken und Schwächen identifizieren.

Fragen 2 und 3 wurden in dem Fragebogen direkt formuliert. Es gab abschließend offene Fragen danach, was die Teilnehmenden an dem Projekt für besonders gelungen gehalten haben, was ihnen gefehlt hat und um welche Elemente das Projekt ergänzt werden sollte.

\subsection{Forschungsergebnisse}

Die Antworten auf die erste Frage, inwiefern das Projekt zur Entwicklung der Lehrkompetenz beigetragen habe, ergaben überwiegend ein positives Bild: $61 \%$ waren entschieden damit einverstanden, dass die fördernde Bewertung eine motivierende Funktion hatte, 33 \% stimmten der Feststellung eher zu. Diese positive Wahrnehmung spiegelt sich in den Kommentaren der Studierenden wieder:

„Die Bewertungen und Kommentare haben mich dazu motiviert, meinen Unterricht besser vorzubereiten" $[\mathrm{Fb} 2]^{26}$.

„Die M einungen der Unterrichtteilnehmenden beeinflussten unsere weiteren Ideen zur Unterrichtsplanung; wenn etwas anerkannt wurde, dann war das eher motivierend“ $[\mathrm{Fb} 6]^{27}$.

Nicht so einig waren sich die Befragten bei der zweiten Frage, ob die Teilnahme am Projekt ihre Selbstsicherheit beim Unterrichten erhöht habe. Nur 28 \% stimmten entschieden zu und 33 \% stimmten eher zu, was die ausgewählten Kommentare widerspiegeln:

${ }^{26}$ "Oceny i komentarze w arkuszach oceny zmobilizowały mnie do tego, żeby lepiej przygotowywać lekcje".

${ }^{27}$ „opinia uczestników przeprowadzonych przez nas zajęć wpływa na korygowanie naszych kolejnych pomysłów dotyczących planowania lekcji; jeśli coś było docenione to raczej było to motywujące" 
„Die Bewertungen und Kommentare haben meine Aufmerksamkeit auf die Schwächen der durchgeführten Unterrichtssequenz gelenkt. Dadurch konnte ich Schlussfolgerungen ziehen und viel lernen. Das wirkte sich positiv auf die Art und Weise aus, wie ich den Unterricht führe" [Fb 8 $]^{28}$.

„Ich weiß, dass mein Unterricht ziemlich gut war. Die Kommentare haben mich in dem Glauben nicht bestärkt" [Fb 7] ${ }^{29}$.

„Die Bewertungen und Kommentare haben meine Selbstsicherheit keinesfalls beeinflusst, denn ich fühle mich darin ohnehin ziemlich sicher" $\left.{ }^{\prime \prime} \mathrm{Fb} \mathrm{2}\right]^{30}$.

Ziel des Projektes war es, qualitative Veränderungen in den Unterrichtsmethoden der Studierenden herbeizuführen und dieses Zel scheint erreicht worden zu sein. Mit der Feststellung "Ich weiß, worauf ich achten soll und was ich in meinen Unterrichtsmethoden verändern soll " waren $61 \%$ entschieden einverstanden und 33 \% stimmten dem eher zu. In einem Fragebogen wurde allerdings festgestellt, die Kommentare würden sich ausschließen, was auf ihre starke Subjektivität verweist [Fb 15] und was bereits an anderen Beispielen gezeigt werden konnte. Es ist deshalb zu betonen, dass jede Evaluation trotz „objektiv“ und gemeinsam festgelegter Beurteilungskriterien subjektive Wahrnehmungen widerspiegelt und deshalb für Verzerrungen anfällig ist. Insofern sollten vereinzelte Kommentare mit einer gewissen Distanz betrachtet werden. Das zeigt aber zugleich das Potenzial von kollegialer Mitbewertung. Sie liefert ein Bild mehrerer subjektiver Wahrnehmungen, die sich an bestimmten Stellen überlappen und somit gewisse Tendenzen zu verzeichnen vermögen.

$28 \%$ stimmten entschieden zu, dass das Projekt innen geholfen habe, ihre Stärken und Schwächen zu identifizieren, während 61 \% dieser Feststellung eher zugestimmt haben:

„Dank der Bewertungen und Kommentare wurde ich mir der Schwächen meiner Unterrichtsmethoden bewusst, die ich persönlich nicht identifizieren und bestimmen konnte. Ich glaube, dass die M einung von Beobachtern für die Verbesserung von Unterrichtsmethoden unumgänglich ist" $[\mathrm{Fb} \mathrm{18}]^{31}$.

${ }^{28}$ "Oceny i komentarze zwróciły moją uwagę na istotne problemy dotyczące prowadzonej lekcji. Dzięki temu mogłam wyciągnąć wnioski i dużo się nauczyć, co przełożyło się na lepszy sposób prowadzenia przeze mnie lekcji".

${ }^{29}$ "Wiem, że lekcja była w miarę dobrze przeprowadzona. Komentarze nie stanowiły podstawy, by mnie w tym utwierdzić".

${ }^{30}$ "Oceny i komentarze nie wpłynęły w żaden sposób na moją samoocenę, ponieważ zawsze czuję się dosyć pewnie kiedy prowadzę lekcję".

31 "Dzięki ocenom i komentarzom mogłam zwiększyć moją świadomość na temat słabych stron moich metod nauczania, których indywidualnie nie byłem w stanie zidentyfikować 
„Während einer Präsentation kann man nicht all seine Stärken und Schwächen präsentieren, deshalb ist das Urteil nicht hundertprozentig adäquat. Es hat mich aber zur Reflexion über Unterrichtsmethoden und Ansätze angeregt" [Fb 13] ${ }^{32}$.

Die Subjektivität der Bewertungen kam mehrmals zur Sprache. Den Studierenden wurde zu Beginn des Projektes klar gemacht, dass bei kollegialer Bewertung diese Subjektivität nicht auszuschließen ist, weil sie als Ausgangspunkt vieler individueller Beobachtungen zustande kommt. Die Studierenden wurden am Anfang des Projektes darüber informiert, dass die Note am Ende des Kurses auch von der kollegialen Bewertung abhängen wird, weil diese einen Anspruch auf höhere Objektivität hat. Ein sehr offener Kommentar zeigt aber, dass das, was als Stärke dieser Bewertungsform gelten sollte, zugleich ihre Schwäche ist.

„Wir haben uns eher kollegial bewertet. Obwohl der Evaluationsbogen anonym war, wollte niemand allzu stark die anderen kritisieren. Ich glaube, dass es mehr Schwächen gab (darauf schließe ich aus der Analyse des von mir durchgeführten Unterrichts)" $[\mathrm{Fb} 8]^{33}$.

Diese Aussage lässt gewisse Zweifel an der Objektivität schriftlicher Bewertungen entstehen, was die Ergebnisse der durchgeführten Untersuchung sicherlich nicht unproblematisch macht. Die Anonymität sollte einen sicheren Raum gewährleisten, in dem konstruktive Kritik zum Ausdruck gebracht werden kann. Der Kommentar indiziert jedoch auf die Befürchtung, den anderen - und im Gegenzug sich selbst - Schaden durch allzu negative Bemerkungen zuzufügen. Über die Berücksichtigung der kollegialen Bewertung als Teil der Abschlussnote muss deshalb in Zukunft noch kritisch nachgedacht werden. Es müssen Strategien konzipiert werden, die die Defizite dieser Beurteilungsform zu überwinden vermögen.

Die Projektteilnehmenden schätzten ihre Kompetenzen, wenn es um die Arbeit mit Lernenden mir besonderen Förderbedürfnissen geht, positiv ein. Der Feststellung „Ich habe erfahren, wie man im Fremdsprachenunterricht mit Schülern mit besonderen Förderbedürfnissen arbeiten kann" stimmten $72 \%$ entschieden und $22 \%$ eher zu. Und obwohl dieser Teil als „der wichtigste

oraz określić. Uważam, że opinia osób trzecich jest niezbędna do udoskonalenia warsztatu pracy nauczyciela".

${ }_{32}^{32}$ "Podczas jednej prezentacji nie zawsze można pokazać wszystkie swoje dobre strony, więc ocena nie jest w $100 \%$ adekwatna, natomiast na pewno skłoniła mnie do refleksji nad sposobami uczenia, podejściu metodach".

${ }_{33}$ "Ocenialiśmy siebie „po koleżeńsku”, mimo że ankieta była anonimowa, to nikt nie chciał zbyt mocno krytykować innych; myślę, że tych słabszych stron było więcej (po własnej analizie przeprowadzonej przeze mnie lekcji". 
Projektteil“ [Fb 6] eingestuft wurde, wurden auch Aspekte genannt, wo die Kompetenzvermittlung zu kurz kam:

„Ich konnte Probleme von Schülern mit besonderen Förderbedürfnissen kennenlernen, würde mir aber einen (eigenen) Workshopteil (dazu) wünschen, weil die Theorie alleine nicht ausreicht, (...)" $[\mathrm{Fb} \mathrm{14}]^{34}$.

„Die Präsentationen zu besonderen Förderbedürfnissen enthielten viele wissenschaftliche und de facto wenige praktische Inhalte, die man sich schwer aneignen konnte. Ich glaube, ich habe von einigen Unterrichtssequenzen und Präsentationen über Schüler mit besonderen Förderbedürfnissen wenig profitiert" [Fb 18 ${ }^{35}$.

Die Entwicklung der eigenen Evaluationskompetenz, die durch die dritte Forschungsfrage erfasst wurde, wurde überwiegend positiv beurteilt. $33 \%$ gaben entschieden zu, gelernt zu haben, fördernde Bewertung anzuwenden. $44 \%$ der Befragten stimmten dieser Feststellung eher zu. $22 \%$ wählten allerdings die Antwort „Ich weiß nicht".

Die Kernziele des Projektes, für die Vorteile der fördernden Bewertung zu sensibilisieren und die Studierenden dazu anzuregen, fördernde Bewertung anzuwenden, scheinen zumindest gewissermaßen erreicht worden zu sein, was mehrere Kommentare widerspiegeln:

"Jetzt weiß ich, worauf genau fördernde Bewertung beruht, ich weiß, wie man sie während des Unterrichts anwendet, deshalb glaube ich, dass der Unterricht in dieser Form sehr vorteilhaft war und mein Wachstum gefördert hat" $[\mathrm{Fb} 4]^{36}$.

„Ich habe gelernt, meine M einungen so zu formulieren, dass sie auch Lösungsvorschläge enthalten und sowohl auf Stärken als auch Schwächen hinweisen“ [Fb 12 ${ }^{37}$.

„Um richtig zu lernen, fördernde Bewertung einzusetzen, sollte man eine komplettere Liste an Kriterien und Fragen schaffen. Man sollte auch die Regel einführen,

\footnotetext{
34 "Tak, mogłam poznać problemy, z jakimi muszą się mierzyć uczniowie ze SPE, jednak przydałaby się do tego część warsztatowa, bo sama teoria to za mało".

${ }^{35}$ "Często prezentacje na temat uczniów ze SPE zawierały dużo naukowych i de facto mało praktycznych treści, do tego w małym stopniu przyswajalnych. Myślę, że mało wyniosłam z niektórych lekcji oraz prezentacji o uczniach z różnymi SPE".

36 "Teraz wiem, na czym dokładnie polega ocenianie kształtujące, wiem jak je stosować podczas lekcji, dlatego uważam, że przeprowadzenie zajęć $w$ takiej formie było bardzo korzystne i znacznie się przyczyniło do mojego wzrostu".

${ }^{37}$ "Nauczyłam się, by moje opinie formułować tak, by zawrzeć propozycję rozwiązania problemu, wskazać zarówno mocne, jak i słabe strony".
} 
dass man keine leeren und nichtssagenden Kommentare wie es war super oder toller Unterricht schreiben darf" [Fb 18] $]^{38}$.

\section{Schlussfolgerungen}

Die Analyse der Forschungsergebnisse zeigt, dass die vorausgesetzten Projektziele nur teilweise erreicht wurden. Die Studierenden konnten eine Menge an Ideen zur Unterrichtsführung austauschen und dadurch ihre eigene Kreativität entfalten. Sie bekamen die Chance, andere zu beobachten sowie ihre Unterrichtskompetenz im sicheren Raum der eigenen Gruppe zu erproben. Umgehend bekamen sie auch praktische Hinweise zur Unterrichtsführung. Den Forschungsergebnissen zufolge wurde ihre Kompetenz, mit Schülern mit besonderen Förderbedürfnissen zu arbeiten, entwickelt. Sie wurden mit den Prinzipien fördernder Bewertung und mit dem Formulieren von konstruktivem Feedback vertraut gemacht, was zw eifelsohne zu den Vorteilen des durchgeführten Projektes gehört.

Trotz vieler positiver Aspekte, die sich in den Kommentaren widerspiegeln, haben lediglich ca. $20 \%$ der Projektteilnehmenden das dreistufige Feedback mit Änderungsvorschlägen angewendet, was kein zufriedenstellendes Ergebnis ist. Nach den M einungen der Studierenden wies das Projekt auch gewisse Defizite auf. Diese M ängel lassen sich in drei Gruppen erfassen:

(1) Vor- und Nachbereitung der Unterrichtssequenzen,

(2) Vermittlung von Informationen zu besonderen Förderbedürfnissen,

(3) Instruktion zum Erteilen von fördernder Bewertung.

Als unzulänglich wurden die Vor- und Nachbereitung der Unterrichtssequenzen betrachtet. Die Studierenden hätten sich mehr Unterstützung seitens der Dozentin bei der Unterrichtsvorbereitung sowie eine zusammenfassende Diskussion nach Beendigung des Projektes gewünscht. Mangelnde Zeit für Diskussionen wurde ebenfalls als eine der Schwächen des Projektes angesehen. Den Teilnehmenden hätten auch Hinweise gefehlt, wie man an seinen Schwächen arbeiten kann. Was den Aspekt der Arbeit mit Lernenden mit besonderen Förderbedürfnissen angeht, so hätten sich die Studierenden eine fertige Sammlung an Aufgaben für Lernende mit besonderen Förderbedürfnissen gewünscht. Thematisiert wurde auch, dass die Unterrichtssequenz nur eine Simulation und keine reale Situation in der Schulklasse darstellte. Es wurde auch angedeutet, dass die Länge der Kommentare am Anfang des Projektes nicht

${ }^{38}$ "...) aby w pełni nauczyć się stosować OK, należałoby stworzyć większą ilość kryteriów i rozbudowanych pytań oraz przyjąć zasadę dotyczącą pisania komentarzy: brak pustych i nic nie wnoszących treści typu: było super, fajna lekcja". 
präzisiert wurde, was dazu geführt hätte, dass sie von sehr unterschiedlicher Qualität und Aussagekraft waren.

Die kritischen Kommentare liefern Impulse zu einem konstruktiven Diskurs, wie dieser Teil des Trainings für angehende Lehrkräfte in Zukunft verlaufen sollte und welche Änderungen vorzunehmen sind, um seine Qualität zu verbessern. Zahlreiche Aussagen der Projektteilnehmenden lassen jedoch die Schlussfolgerung zu, dass durch das Projekt die Reflexions- sowie Evaluationskompetenz der angehenden Fremdsprachenlehrenden entwickelt wurde. Die Forschungsergebnisse bestätigen auch die Notwendigkeit, fördernde Bewertung zum inhärenten Teil des Lehrertrainings zu machen. Wie Komorowska (2018: 196) aufgrund der Analyse einschlägiger Literatur feststellt, zeigen Lehrer mehr Bereitschaft, fördernde Bewertung einzusetzen, wenn sie selbst während ihrer Ausbildungszeit oder während der Fortbildungen konstruktives und motivierendes Feedback erlebt haben. Die Prinzipien und Vorteile dieser Evaluationsform sollen aber noch stärker ins Bewusstsein angehender Lehrkräfte gerückt und einem noch intensiveren Training unterzogen werden.

\section{LITERATURVERZEICHNIS}

Al-Khamisy D. (2013), Edukacja właczajqca edukacjq dialogu. W poszukiwaniu modelu edukacji dla ucznia ze specjalnymi potrzebami edukacyjnymi. Warszawa: Wydawnictwo Akademii Pedagogiki Specjalnej.

Bohl T. (2004), Prüfen und Bewerten im Offenen Unterricht. Weinheim und Basel: Beltz Verlag.

Bortz J., Döring N. (2003), Forschungsmethoden und Evaluation für Humanund Sozialwissenschaftler. Berlin - Heidelberg - New York: Springer.

Czaplikowska R., Kubacki D. (2019), Kleines Fachlexikon der DaF-Didaktik. Theorie und Unterrichtspraxis. Chrzanów, Kraków: Kubart.

Hattie J. (2015), Widoczne uczenie się dla nauczycieli. Jak maksymalizować siłę oddziaływania na uczenie się. Warszawa: Centrum Edukacji Obywatelskiej. Hattie J., Timperley H. (2007), The power of feedback. "Review of Educational Research", Nr. 1(77), S. 81-112. Online: https://ctl.univie.ac.at/fileadmin/ user_upload/z_ctl/Feedback/Hattie_Timperley_2007_Power_of_Feed back_1_.pdf [ābgerufen am 18.03.2017].

Janicka M. (2017), Umgang mit Diversität im Klassenraum - Annahmen und Realität. „Glottodidactica”, Vol. XLIV/1(2017), S. 9-21.

Janicka M. (2016), Nauczyciele języków obcych wobec różnorodności w klasie językowej oraz wyzwań dydaktyki włączajq̨cej. „Języki Obce w Szkole”, Nr 4, S. 39-46. 
Komorowska H. (2018), Feedback in language learning and teaching. „Glottodidactica", Vol. XLV/2(2018), S. 185-199.

Kordziński J. (2013), Nauczyciel, trener, coach. Warszawa: ABC. Wolters Kluwer Business.

Reich K. (2004), Konstruktivistische Didaktik. Lehren und Lernen aus interaktionistischer Sicht. M ünchen, Unterschleißheim: Luchterhand.

Reich K. (2014), Inklusive Didaktik. Bausteine für eine inklusive Schule. Weinheim und Basel: Beltz Verlag.

Siebert H. (2005), Pädagogischer Konstruktivismus. Lernzentrierte Pädagogik in Schule und Erwachsenenbildung. Weinheim und Basel: Beltz Verlag.

Sterna D. (2016), Uczę się uczyć. Ocenianie kształtujqce w praktyce. Warszawa: Centrum Edukacji Obywatelskiej.

Ziebell B. (2007), Unterrichtsbeobachtung und Lehrerverhalten. Fernstudieneinheit 32. Videosequenzen. Berlin: Langenscheidt.

\section{INTERNETQUELEN}

http://prawo.sejm.gov.pl/isap.nsf/download.xsp/WDU20120000131/0/D20 120131.pdf [abgerufen am 08.10.2019]

http:// isap.sejm.gov.pl/isap.nsf/download.xsp/WDU20190001450/0/D2019 1450.pdf [abgerufen am 08.10.2019] 